\title{
The accuracy of a novel pedicle screw insertion technique assisted by a special angular scale in the subaxial cervical spine using lateral mass as a reference marker
}

Hang Shi ${ }^{1+}$, Lei Zhu ${ }^{1 \dagger}$, Jun Ma², Yu-Cheng Zhu² and Xiao-Tao Wu ${ }^{1 *}$

\begin{abstract}
Background: Posterior cervical pedicle screw (CPS) internal fixation has better biomechanical stability than other posterior cervical fixation methods. However, this technique is limited in clinical practice due to the complex anatomical structure and the adjacent relationship of the cervical pedicle, and the high risk of neurovascular injury. The purpose of this study was to describe a novel subaxial CPS insertion technique assisted by a special angular scale using lateral mass as a reference marker and to evaluate the accuracy of CPS placement and the distribution characteristics of CPS misplacement.
\end{abstract}

Methods: A total of 36 patients with subaxial cervical spine diseases who underwent posterior CPS fixation were consecutively selected. The optimal entry point on the posterior surface of the lateral mass was identified on the three-dimensional cervical model reconstructed from preoperative computed tomography $(\mathrm{CT})$ images. The pedicle transverse angle (PTA) and pedicle-lateral mass angle (PLMA) were measured on the transverse and sagittal CT images respectively. The pedicle screws were inserted according to the preoperatively planned entry point and angles. We analysed the postoperative CT images for CPS misplacement rates and perforation directions following the Lee classification.

Results: Overall, 177 pedicle screws were inserted, of which 119 (67.2\%) were classified as grade 0,43 (24.3\%) as grade $1,12(6.8 \%)$ as grade 2 and $3(1.7 \%)$ as grade 3 by the postoperative CT images. The accuracy rate of CPS placement was 91.5\%. Of the 15 misplaced pedicle screws (grades 2 and 3), 11 were lateral pedicle perforations, 3 were superior perforations and 1 was an inferior perforation. There were no neurovascular injuries related to CPS misplacement.

Conclusions: With our technique, the optimal entry point and two angles (PTA and PLMA) were identified for CPS insertion. The novel CPS insertion technique assisted by a special angular scale provides high accuracy and few complications.

Keywords: CPS, Special angular scale, Lateral mass, Optimal entry point, PTA, PLMA

\footnotetext{
* Correspondence: wuxiaotaospine@seu.edu.cn

'Hang Shi and Lei Zhu contributed equally to this work.

'Department of Spine Surgery, Zhongda Hospital, School of Medicine,

Southeast University, Nanjing 210009, Jiangsu, China

Full list of author information is available at the end of the article
}

(c) The Author(s). 2020 Open Access This article is licensed under a Creative Commons Attribution 4.0 International License, which permits use, sharing, adaptation, distribution and reproduction in any medium or format, as long as you give appropriate credit to the original author(s) and the source, provide a link to the Creative Commons licence, and indicate if changes were made. The images or other third party material in this article are included in the article's Creative Commons licence, unless indicated otherwise in a credit line to the material. If material is not included in the article's Creative Commons licence and your intended use is not permitted by statutory regulation or exceeds the permitted use, you will need to obtain permission directly from the copyright holder. To view a copy of this licence, visit http://creativecommons.org/licenses/by/4.0/ The Creative Commons Public Domain Dedication waiver (http://creativecommons.org/publicdomain/zero/1.0/) applies to the data made available in this article, unless otherwise stated in a credit line to the data. 


\section{Introduction}

With the development of cervical surgery techniques, posterior cervical internal fixation has been widely applied in the treatment of various subaxial cervical spine diseases. The commonly used subaxial cervical posterior screw fixation methods include pedicle screw, lateral mass screw, lamina screw and transfacet screw [1-3]. Many studies have reported that the cervical pedicle screw (CPS) method has better biomechanical stability than other posterior cervical fixation methods [1, 4]. Nevertheless, there are potential risks of injury to the surrounding neurovascular tissues, such as the spinal cord, vertebral arteries and nerve roots, as a result of CPS misplacement due to the anatomical variability of the cervical pedicle, a small pedicle size or a large pedicle transverse angle (PTA) [5-7].

A variety of CPS insertion strategies have been advocated to improve the accuracy of screw placement and avoid neurovascular injury complications. Some innovative techniques, such as navigation systems, are restricted because of the high cost and the added procedure time [8]. Therefore, freehand CPS insertion techniques are still widely used in clinical practice [9]. In this study, we describe a novel freehand CPS insertion technique assisted by a special angular scale using lateral mass as a reference marker and evaluate the accuracy of CPS placement and the distribution characteristics of CPS misplacement in patients with various subaxial cervical spine diseases.

\section{Materials and methods}

\section{Patient population}

From January 2014 to December 2018, 36 patients with subaxial cervical spine diseases who underwent the posterior cervical pedicle fixation using the novel CPS insertion technique were consecutively selected. There were 23 males and 13 females with an average age of 53 years (range 27-75 years). The diagnoses included trauma (6 patients), cervical spondylotic myelopathy and spinal stenosis (15 patients), ossification of the posterior longitudinal ligament (9 patients) and spinal cord tumour (6 patients). The study was performed in compliance with ethical standards and was approved by the institutional review board of our hospital. The inclusion criteria were patients aged at least 18 years, no previous cervical spine surgery and clear images from the third through seventh cervical vertebrae. Patients with infectious, cervical vertebral pedicle damage, cervical vertebral rotation, congenital cervical deformity or anatomical variation were excluded from the study. The follow-up methods involved outpatient service, e-mail and telephone call surveys to assess postoperative recovery and determine whether any neurovascular injury or other complications related to CPS misplacement developed.

\section{Preoperative examination}

All patients underwent preoperative radiography, computed tomography (CT) and magnetic resonance imaging (MRI) scans. Multiplanar reconstruction of the target cervical vertebra was performed by the transverse CT images.

The ideal pedicle trajectory was defined as a line passing through the centre of the pedicle on the transverse and sagittal CT images. The three-dimensional cervical model was reconstructed using cervical spine transverse CT images. On the three-dimensional reconstructed cervical model, the ideal pedicle trajectory would penetrate the posterior surface of the lateral mass, and this point was then identified as the optimal entry point passing through the centre of the pedicle (Fig. 1). Vertical and horizontal offsets of the optimal entry point were measured from the centre of the lateral mass (Fig. 2). The preoperative PTA and pediclelateral mass angle (PLMA) were measured on the transverse and sagittal CT images, respectively, and other parameters, including the pedicle width, pedicle height and pedicle axis length, were also measured (Fig. 3). The PTA referred to the included angle between the pedicle trajectory and the vertical line of the posterior edge of the vertebral body on the transverse CT images, and the PLMA was defined as the included angle between the pedicle trajectory and the posterior edge of lateral mass on the sagittal CT images. The PLMA on the specimen was shown in Fig. 4.

\section{Surgical procedure}

All patients were placed in the prone position with their skull fixed using a Mayfield clamp. Ensure that the cervical spine process was in the middle and there was no lateral tilt. A straight posterior midline incision was made. The cervical lamina and lateral mass were fully exposed by dissecting the paravertebral muscles during the operation (Fig. 5). The centre of the lateral mass was marked, and the optimal entry point on the posterior surface of the lateral mass was then identified following the preoperative measurements of the vertical and horizontal offsets. An entry hole was made by using a power drill at the optimal entry point (Fig. 6) and the pedicle canal was created with a special angular scale combined with a wire tapping according to the preoperative measurements of the PTA and PLMA (Fig. 7). The detailed processes were as follows: during the operation, the reference axis was placed parallel to the spinous processes of the cervical spine, and then the adjusting spindle was regulated to achieve the preset PTA. To control PLMA as accurately as possible in clinical practice, after identifying the entry hole, the wire tapping inside the adjusting spindle was first placed perpendicular to the lateral mass and then slightly adjusted to the head or tail side 


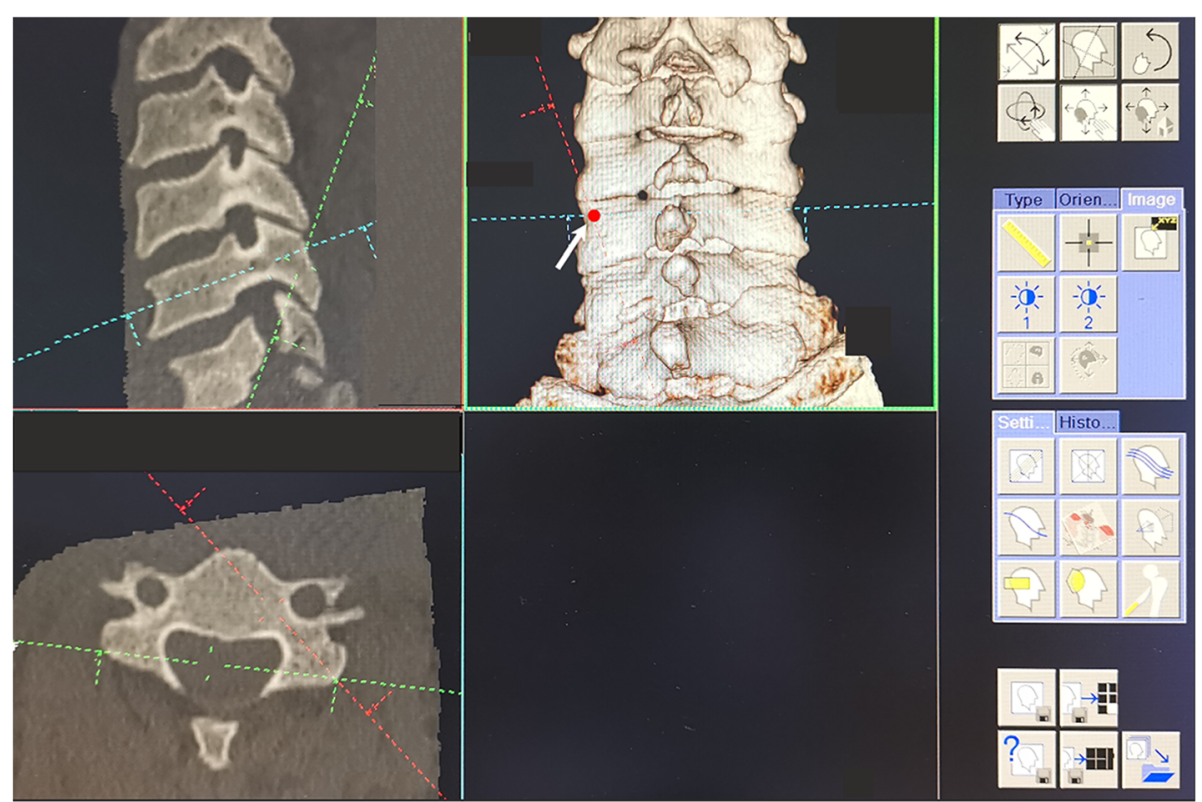

Fig. 1 The ideal pedicle trajectory was defined as a line passing through the centre of the pedicle on the transverse and sagittal CT images (left column). The three-dimensional cervical model was reconstructed using cervical spine transverse $C T$ images. On the three-dimensional reconstructed cervical model, the ideal pedicle trajectory would penetrate the posterior surface of the lateral mass, and this point was then identified as the optimal entry point passing through the centre of the pedicle (right column)

according to the preset PLMA. The pedicle canal was ascertained with a probe to ensure its safety, and then, a pedicle screw with a diameter of $3.5 \mathrm{~mm}$ and a length of 20-24 mm was inserted. Laminectomy, laminoplasty or tumour removal were performed for posterior

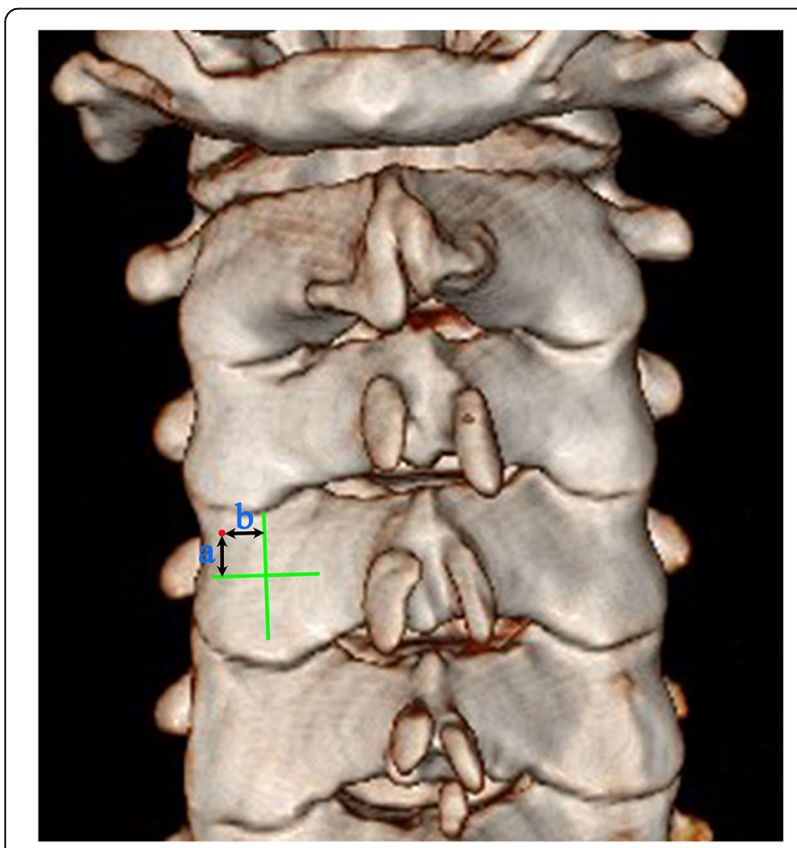

Fig. 2 Vertical and horizontal offsets of the optimal entry point $(\mathbf{a}, \mathbf{b})$ were measured from the centre of the lateral mass decompression. Finally, the rod with the appropriate size was bent in accordance with normal cervical curvature and the connections between the screws and rod were tightened (Fig. 8).

\section{Postoperative imaging evaluation of CPS placement}

A postoperative CT scan and multiplanar reconstruction of the fixed segments were performed, and the CT images were assessed by an independent radiologist and an experienced spine surgeon who did not perform the surgery and was blind to the surgery. The accuracy of CPS placement was evaluated according to the Lee classification [10]: grade 0 , no perforation; grade 1 , perforation less than $25 \%$ of the screw diameter; grade 2 , perforation from $25 \%$ to $50 \%$ of the screw diameter; and grade 3 , perforation more than $50 \%$ of the screw diameter. CPSs with grades 0 and 1 were regarded as accurately placed screws, and those with grades 2 and 3 were regarded as misplaced screws. The directions of pedicle perforation were classified into four categories: inferior, superior, medial and lateral.

\section{Postoperative complications analysis}

Postoperative complications such as spinal cord injury, vertebral artery injury, nerve root injury or internal fixation failure resulting from CPS misplacement were recorded and analysed. 

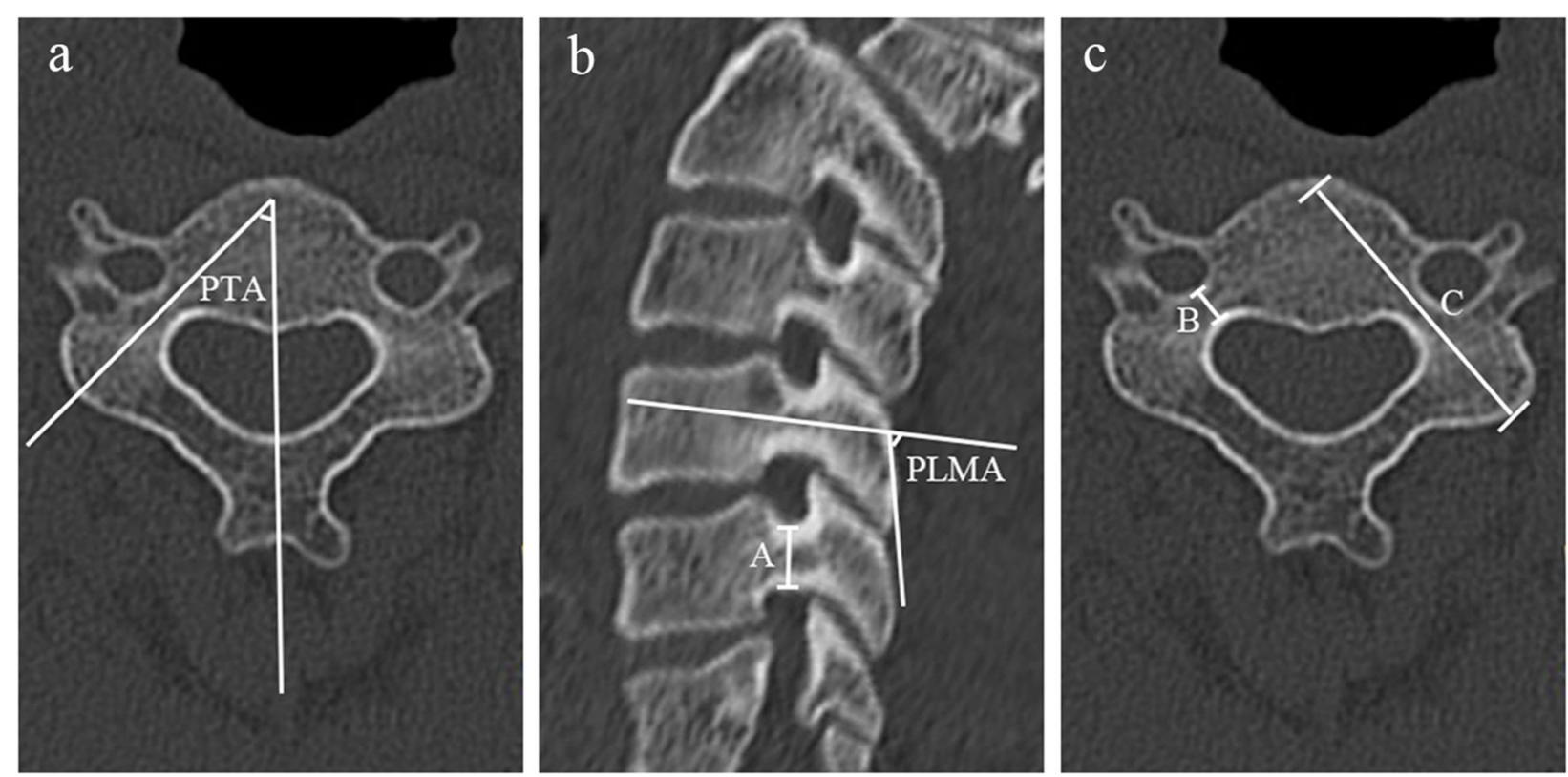

Fig. 3 The preoperative pedicle transverse angle (PTA) and pedicle-lateral mass angle (PLMA) were measured on the transverse and sagittal CT images, respectively $(\mathbf{a}, \mathbf{b})$, and other parameters, including the pedicle height (A), pedicle width (B) and pedicle axis length (C), were also measured $(\mathbf{b}, \mathbf{c})$. The PTA referred to the included angle between the pedicle trajectory and the vertical line of the posterior edge of the vertebral body on the transverse CT images, and the PLMA was defined as the included angle between the pedicle trajectory and the posterior edge of lateral mass on the sagittal CT images

\section{Statistical analysis}

All data were analysed using SPSS Statistics software (version 22.0, SPSS Inc, Chicago, IL, USA). Continuous variables were summarised using mean \pm standard deviation (SD). The differences in the preoperative angles of two sides were analysed using $t$ test. The differences in the CPS misplacement rates and perforation directions were analysed using Fisher's exact test, with $P<0.05$ considered to be statistically significant.

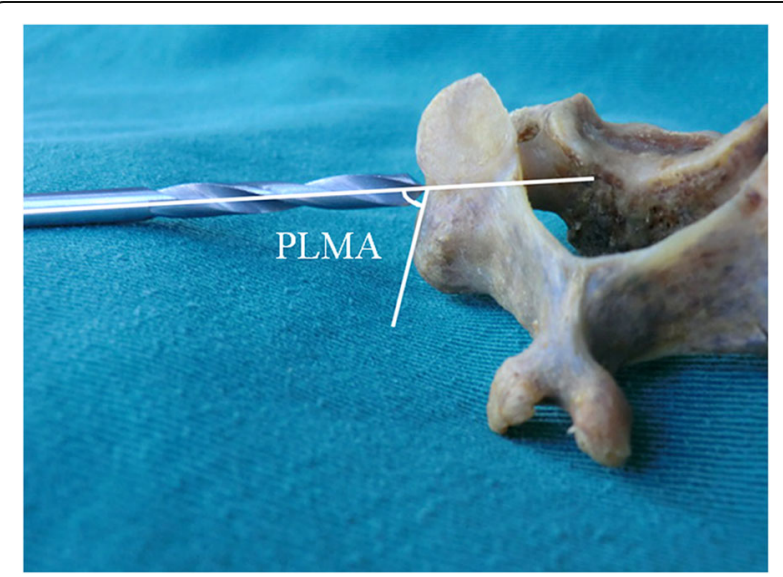

Fig. 4 The diagram of the pedicle-lateral mass angle (PLMA) on a specimen

\section{Results}

All patients underwent the surgeries successfully. There were 5 incidences of conversion to lateral mass screws due to muscle obstruction. A total of 177 pedicle screws were inserted, including 90 on the left and 87 on the right. A total of 112 and 65 pedicle screws were inserted into male and female patients, respectively. The measurements of preoperative PTA and PLMA are shown in Table 1. There was no significant difference between the two sides of each pedicle $(P>0.05)$. The PTA has a decreasing trend from $\mathrm{C} 3$ to $\mathrm{C} 7$, ranging from $25^{\circ}$ to $51^{\circ}$. However, the PLMA has a trend of increasing from C3 to $\mathrm{C} 7$, ranging from $68^{\circ}$ to $103^{\circ}$. The segmental distribution and classification of all pedicle screws are shown in Table 2. Of 177 pedicle screws, 119 (67.2\%) were classified as grade 0 (no perforation), $43(24.3 \%)$ as grade 1 (perforation less than $25 \%$ of the screw diameter), 12 (6.8\%) as grade 2 (perforation between 25 and $50 \%$ of the screw diameter) and $3(1.7 \%)$ as grade 3 (perforation more than $50 \%$ of the screw diameter). The accuracy rate of CPS placement was 91.5\% (162/177) (Fig. 9) and the misplacement rate was $8.5 \%(15 / 177)$.

The perforation directions of the misplaced pedicle screws are shown in Table 3. Of the 15 misplaced pedicle screws (grades 2 and 3), 11 were lateral wall perforations, 3 were superior wall perforations, 1 was an inferior wall perforation and there were no medial wall perforations. The screws with perforations of the lateral 


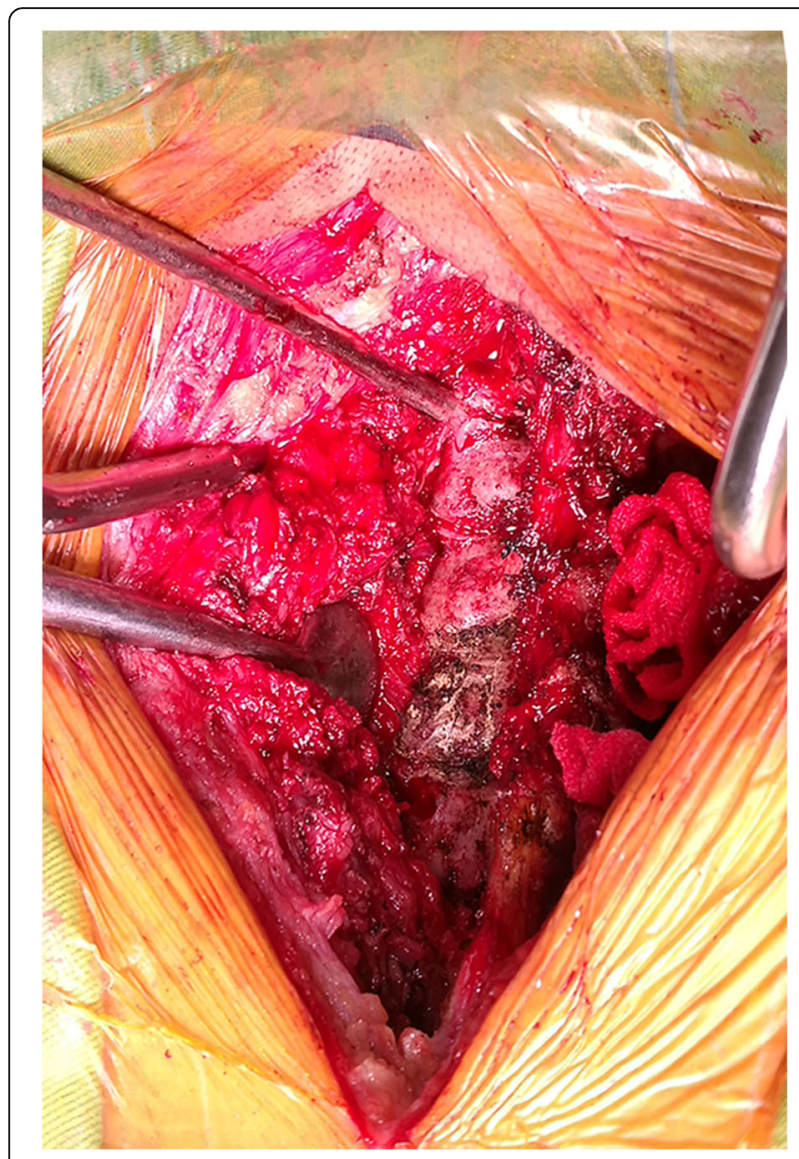

Fig. 5 The lateral masses of target segments were clearly exposed during operation

wall accounted for $73.3 \%(11 / 15)$ of all the misplaced screws, and this rate was higher than that of the superior wall $(20 \%, 3 / 15)$, the inferior wall $(6.7 \%, 1 / 15)$ and the medial wall $(0,0 / 15)$. There were 9 and 6 misplaced screws on the left and right sides, respectively. There was no significant difference between the two sides $\left(X^{2}\right.$ $=0.55, P=0.46)$. There were 11 and 4 misplaced screws in male and female patients, respectively. There was no significant difference between female and male patients $\left(X^{2}=0.71, P=0.40\right)$.

One patient with cervical trauma and quadriplegia died of pulmonary infection and respiratory failure 1 month after the operation and was lost during followup. The other patients were followed up for 12-28 months (mean of 18 months). None of the patients had neurovascular injuries related to CPS misplacement.

\section{Discussion}

Posterior CPS internal fixation has the advantages of strong pull-out resistance and excellent biomechanical stability in the treatment of cervical fractures, tumours, ossification of posterior longitudinal ligament and other

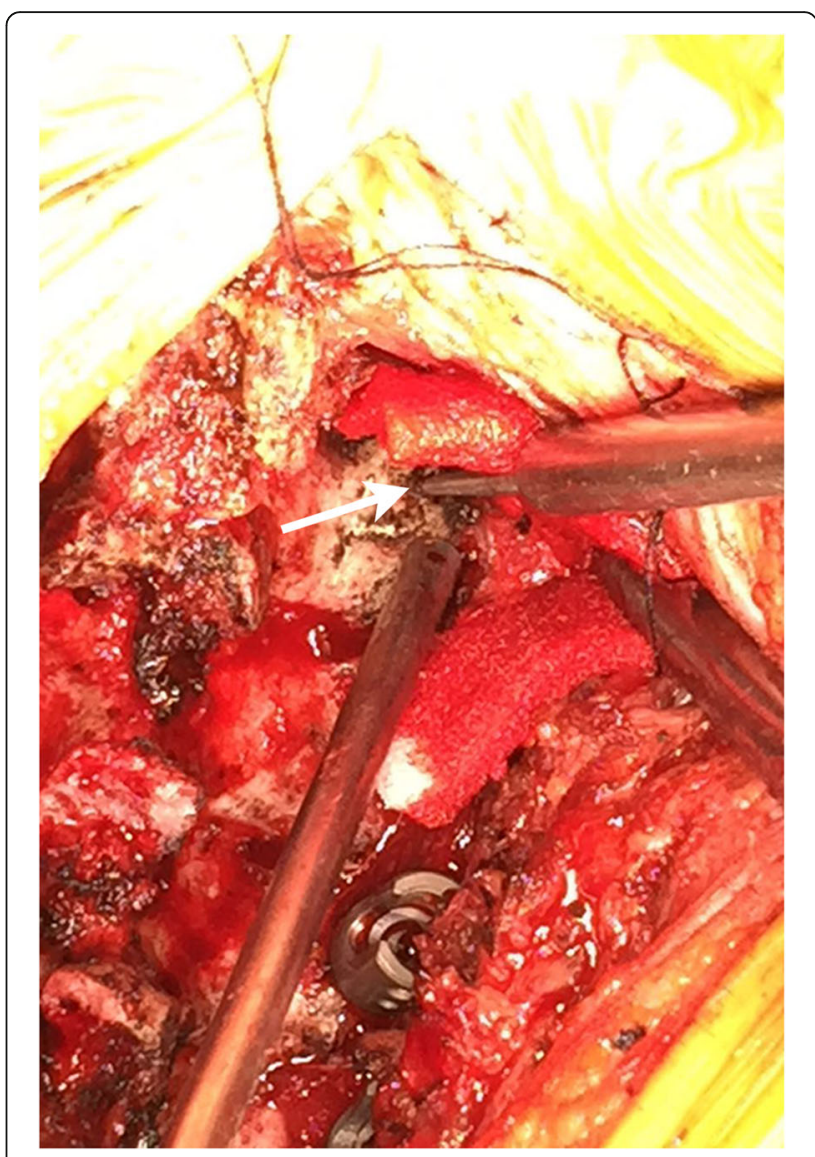

Fig. 6 The entry hole was made by using a power drill at the optimal entry point on the posterior surface of the lateral mass

cervical spine diseases [11]. However, this technique is limited in clinical practice due to the complex anatomical structure and the adjacent relationship of the cervical pedicle, and the high risk of vascular, nerve root and spinal cord injury. Studies have shown that the incidence of complications related to screw misplacement is high, and some of these complications can result in serious consequences $[12,13]$. At present, the technology commonly used for CPS placement mainly includes freehand screw placement technology, computer-assisted navigation technology and 3D-guided template technology. The latter two technologies can improve the safety and accuracy of CPS placement [14, 15]. However, computer-assisted navigation technology has the disadvantages of requiring expensive equipment, a long operative time and a high radiation dose [16]. 3D-guided template technology requires complex preoperative designs, so it is not suitable for emergency surgery patients. Therefore, freehand screw placement technology is still widely applied in clinical practice.

Many former surgeons $[17,18]$ measured the included angle between the upper endplate of the vertebral body and the central axis of the pedicle on the sagittal CT 


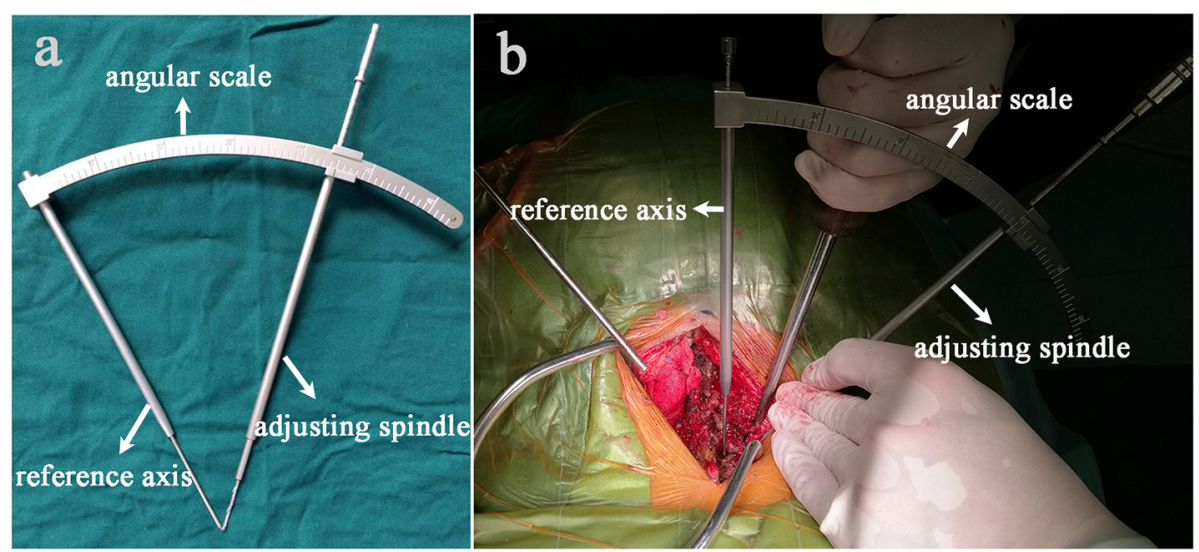

Fig. 7 A special angular scale that controls the angle (a). The pedicle screw was inserted according to preoperative measurements of pedicle transverse angle (PTA) and pedicle-lateral mass angle (PLMA) (b). The detailed processes were as follows: during the operation, the reference axis was placed parallel to the spinous processes of the cervical spine, and then the adjusting spindle was regulated to achieve the preset PTA. To control PLMA as accurately as possible in clinical practice, after identifying the entry hole, the wire tapping inside the adjusting spindle was first placed perpendicular to the lateral mass and then slightly adjusted to the head or tail side according to the preset PLMA

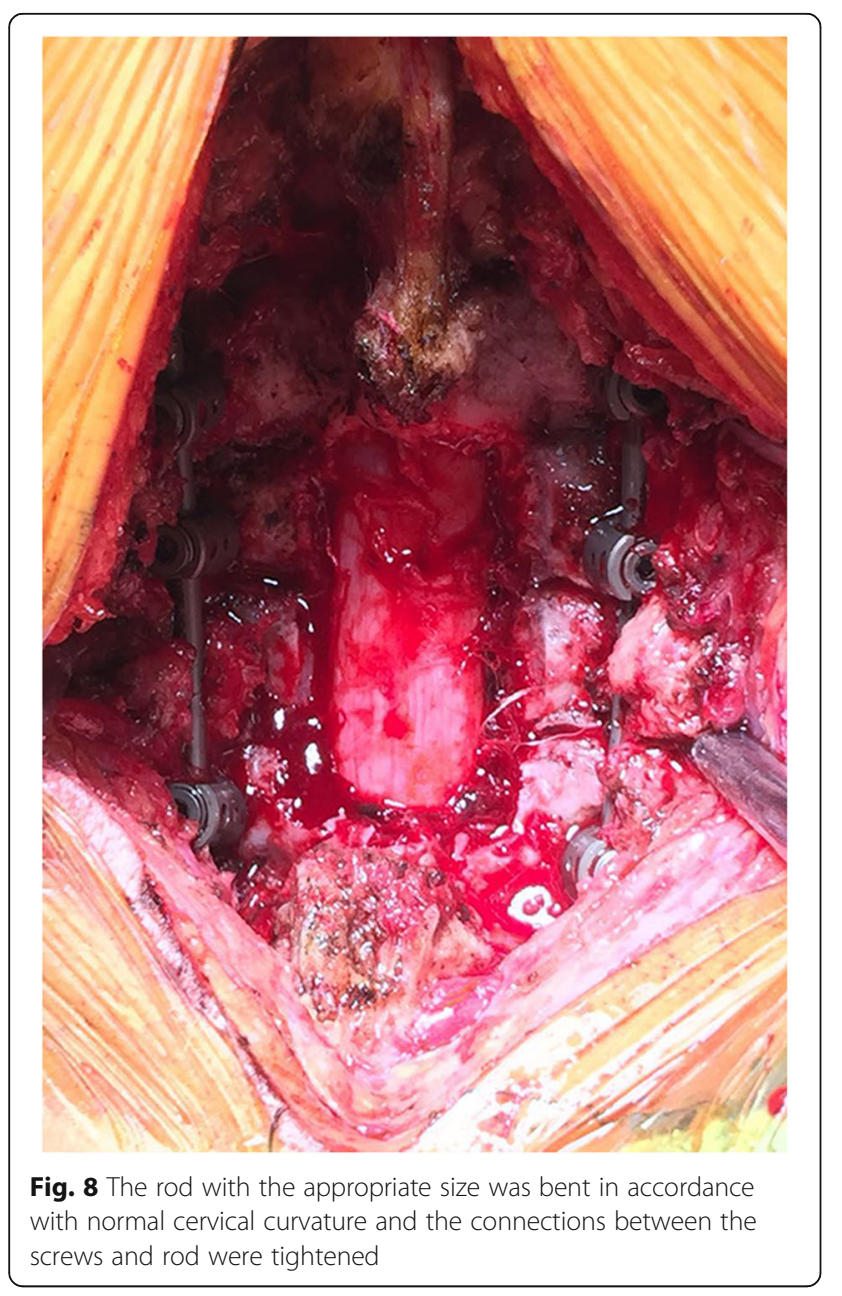

images before surgery and used this measurement for the sagittal plane angle for pedicle screw placement. Then the pedicle screw was placed in accordance with the preoperative measurement angle. Patients commonly underwent preoperative CT scans with the cervical vertebra in the neutral position. However, patients were placed in the prone position during the operation. The cervical curvature cannot reach an agreement between preoperative and intraoperative position, especially for patients with cervical instability or deformity. The preoperative and intraoperative sagittal angle error may increase the risk of screw misplacement and related complications. The intraoperative insertion angle during the process of CPS insertion should be the same as that measured before the operation. The ideal reference plane and angle of screw insertion should not change with the patient position. Therefore, the posterior surface of the lateral mass, which was a visible and constant reference, was used as a reference marker for the sagittal plane angle for CPS insertion. Then, the PLMA was measured, which did not change with the changes in cervical flexion and extension. In the study, the PTA and PLMA were measured on the transverse and sagittal CT images, respectively. The optimal entry point on the posterior surface of the lateral mass was also identified on the three-dimensional cervical model reconstructed before surgery. The CPS was placed with the same reference marker, entry point and angles during the operation. The accuracy rate of CPS placement was 91.5\% (162/177), which was higher than the accuracy (84.9\%) reported by Lee et al. [10]. The results of our research showed that the misplacement rate of CPS placement can be reduced by the novel pedicle screw 
Table 1 Measurements of the Preoperative PTA and PLMA

\begin{tabular}{llllll}
\hline Pedicle & \multicolumn{1}{l}{ PTA $\left(^{\circ}\right)$} & & & PLMA $\left(^{\circ}\right)$ \\
\cline { 2 - 3 } \cline { 5 - 6 } & Left & Right & & Left & Right \\
\hline C3 & $44.8 \pm 3.1(40-50)$ & $43.9 \pm 2.8(39-49)$ & & $77.8 \pm 4.3(72-86)$ & $77.5 \pm 4.5(68-85)$ \\
C4 & $43.2 \pm 3.4(37-48)$ & $43.5 \pm 3.6(39-51)$ & & $79.1 \pm 3.7(74-89)$ & $79.6 \pm 6.0(72-93)$ \\
C5 & $42.3 \pm 4.0(33-51)$ & $42.6 \pm 3.1(38-49)$ & & $83.3 \pm 4.4(76-94)$ & $82.8 \pm 4.6(78-96)$ \\
C6 & $38.7 \pm 4.4(31-45)$ & $38.7 \pm 4.3(29-47)$ & & $87.6 \pm 6.2(78-101)$ & $86.7 \pm 4.7(82-102)$ \\
C7 & $33.9 \pm 3.7(25-42)$ & $35.2 \pm 2.7(29-40)$ & & $90.3 \pm 5.3(80-102)$ & $89.7 \pm 5.2(83-103)$ \\
\hline
\end{tabular}

Data are presented as mean \pm standard deviation

PTA pedicle transverse angle, PLMA pedicle-lateral mass angle

insertion technique assisted by a special angular scale using lateral mass as a reference marker.

The directions of pedicle perforation were classified into four categories: inferior, superior, medial and lateral. In this study, screws with perforations of the pedicle lateral wall accounted for $73.3 \%(11 / 15)$ of all the misplaced screws, and this rate was higher than those of the other three walls; this result is consistent with the results reported by Park et al. [6]. In addition to the fact that the bony cortex of the pedicle lateral wall is thin, we believe that the following factors are also the reasons that the screw penetrated the pedicle lateral wall easily. First, the PTA of the subaxial cervical spine is large, measuring up to $52^{\circ}$ [19]. Due to the obstruction of soft tissues, such as paravertebral muscles, the inclination angle of the screw cannot be sufficiently large during CPS insertion, especially for $\mathrm{C} 3-\mathrm{C} 5$ pedicle screw placement [20]. In addition, for some patients with cervical spine injury or cervical instability, although there is no obvious rotation of the cervical vertebra in the preoperative $\mathrm{CT}$ scans, the cervical vertebra may rotate toward the opposite side due to the stress of drilling during the screw placement process, which is also one of the reasons for the perforation of the lateral wall. Sugimoto et al. [21] reported that 76 pedicle screws were placed in 17 patients with cervical spine injury, and the vertebral rotation was measured during screw insertion. The average degree of cervical vertebral rotation was $9.1^{\circ}$ at $\mathrm{C} 3,7.8^{\circ}$ at $\mathrm{C} 4,6.7^{\circ}$ at $\mathrm{C} 5,4.9^{\circ}$ at $\mathrm{C} 6$ and $2.8^{\circ}$ at $\mathrm{C} 7$. In our study, the assistant used a forcep to temporarily immobilise the spinous process of the cervical spine during the screw

Table 2 Segmental distribution and classification of 177 pedicle screws in 36 patients

\begin{tabular}{llllll}
\hline Grade & C3 & C4 & C5 & C6 & C7 \\
\hline 0 & 12 & 19 & 24 & 35 & 29 \\
1 & 7 & 8 & 13 & 9 & 6 \\
2 & 3 & 4 & 3 & 1 & 1 \\
3 & 1 & 1 & 1 & 0 & 0 \\
Total & 23 & 32 & 41 & 45 & 36 \\
\hline
\end{tabular}

placement process to avoid cervical vertebral rotation. Besides, the screws with perforations of the superior and inferior wall accounted for $26.7 \%(4 / 15)$ of all the misplaced screws in our study. This may be due to an error between the actual PLMA and the preset PLMA during the operation, which further resulted in the tilt of the pedicle screws to the cephalic or caudal sides.

Not all misplaced screws cause complications. In a study reported by Nakashima et al. [12], 390 cervical pedicle screws were inserted into 84 patients and 76 of these pedicle screws were misplaced. The rate of misplacement was $19.5 \%$. Complications related to screw misplacement occurred in 5 patients, including 3 patients with nerve root injury and 2 patients with vertebral artery injury. In a study described by Yoshihara et al. [22], 2668 subaxial cervical pedicle screws were inserted into 661 patients. The incidence of vertebral artery injury was $0.61 \%(4 / 661)$, and the incidence of nerve root injury was $0.31 \%(2 / 661)$. In our study, a total of 177 pedicle screws were inserted into 36 patients, and 15 of these pedicle screws were misplaced. However, there were no neurovascular injuries related to screw misplacement.

Although the screw placement technique proposed in this study has high accuracy and few complications, it also has some limitations. First, the key to the technique is to determine one optimal entry point and two angles (PTA and PLMA). The PTA is defined as the included angle between the central axis of the pedicle and the vertical line of the posterior edge of the vertebral body on the transverse CT images. However, for some patients with a cervical rotatory deformity or articular interlocking caused by cervical spine injury, the vertical line of the posterior edge of the vertebral body also rotates synchronously, which leads to preoperative and intraoperative PTA errors and increases the risk of the pedicle screw penetrating the medial or lateral wall. Second, for some patients with a cervical deformity or an anatomical structure variation of the lateral mass, the included angle between the central axis of the pedicle and the posterior surface of the lateral mass cannot be accurately 

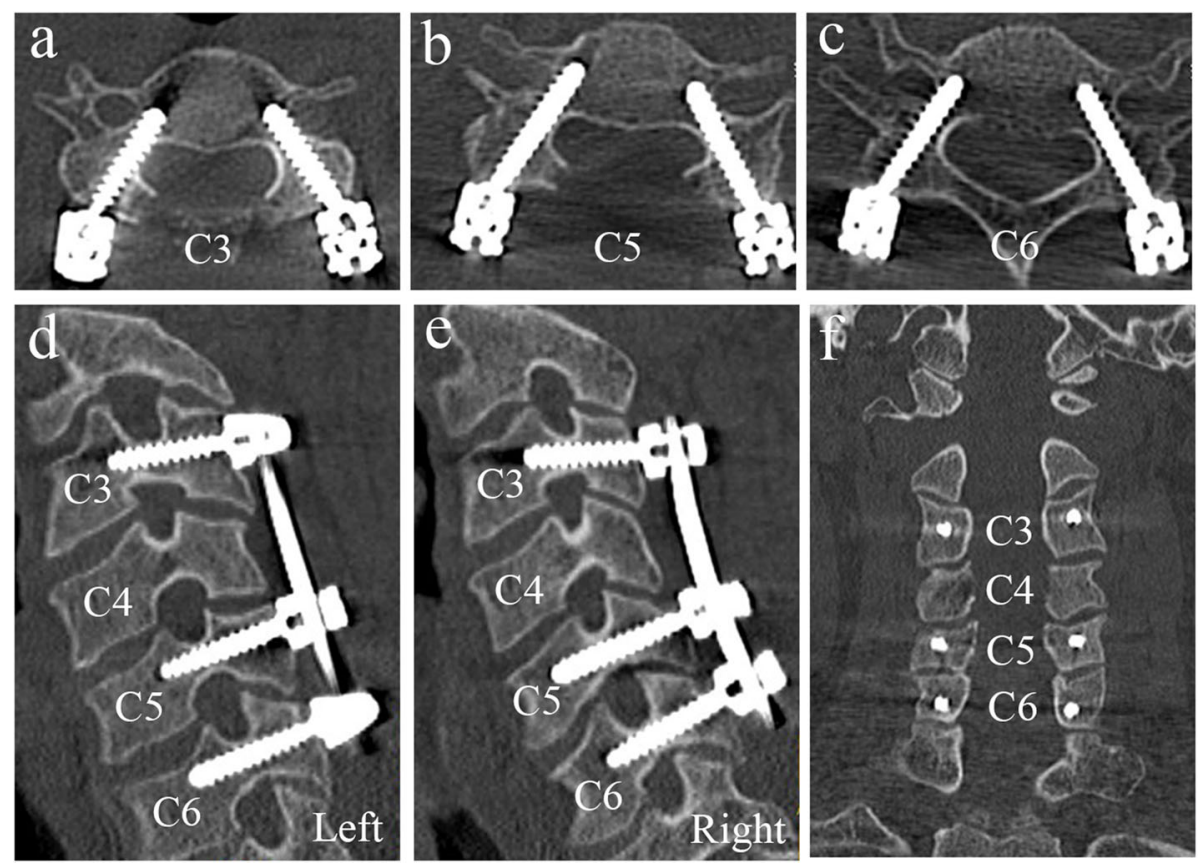

Fig. 9 A typical case with successful cervical pedicle screw (CPS) fixation. Postoperative CT scans and multiplanar reconstruction showed the good placement of pedicle screws at C3, C5, C6 levels (a, b, c, d, e, f)

measured, and the optimal entry point on the posterior surface of the lateral mass cannot be precisely identified. Third, in this study, PTA can be precisely controlled by the special angular scale, but PLMA cannot be precisely quantified. We can find that the PLMA is getting closer and closer to $90^{\circ}$ from C3 to C7 (Table 1). To control PLMA as accurately as possible in the clinical practice of this study, after identifying the entry hole, the wire tapping inside the adjusting spindle was first placed perpendicular to the lateral mass and then slightly adjusted to the head or tail side according to preoperative measurement PLMA. Furthermore, compared with the research results of other scholars, there are some errors in the accuracy of screw placement because there is no uniform standard for screw misplacement.

Table 3 Perforation directions of 15 misplaced pedicle screws

\begin{tabular}{lllll}
\hline Pedicle & Lateral $^{*}$ & Superior & Inferior & Medial \\
\hline C3 & 4 & 0 & 1 & 0 \\
C4 & 2 & 1 & 0 & 0 \\
C5 & 3 & 1 & 0 & 0 \\
C6 & 1 & 0 & 0 & 0 \\
C7 & 1 & 1 & 0 & 0 \\
Total & 11 & 3 & 1 & 0 \\
\hline
\end{tabular}

*Statistically significant using chi-squared tests $P<0.05$

\section{Conclusions}

This study shows results for a novel CPS insertion technique assisted by a special angular scale using the lateral mass as a reference marker. With our technique, the optimal entry point and two angles (PTA and PLMA) are identified before the operation, and the screws are inserted with the same entry point and angles during the operation. The novel CPS insertion technique provides high accuracy and few complications.

\section{Supplementary Information}

The online version contains supplementary material available at https://doi. org/10.1186/s13018-020-02054-1.

\section{Additional file 1.}

\section{Abbreviations}

CPS: Cervical pedicle screw; CT: Computed tomography; PTA: Pedicle transverse angle; PLMA: Pedicle-lateral mass angle; MRI: Magnetic resonance imaging

\section{Acknowledgements \\ Not applicable.}

\section{Authors' contributions}

HS, LZ and XTW designed the study. HS, JM and YCZ collected the data. LZ and JM performed the statistical analysis and interpreted the results. HS, LZ and XTW drafted the manuscript. The final manuscript was approved by all authors. 


\section{Funding}

This study was funded by the Graduate Student Scientific Research Innovation Projects of Jiangsu Province (KYCX18_0176), the Fundamental Research Funds for the Central Universities (2242019 K40257), and Suqian Science and Technology Bureau (S201806).

\section{Availability of data and materials}

All data generated or analysed during this study are included in its supplementary information files.

\section{Ethics approval and consent to participate}

This study was approved by the Institutional Review Committee of Zhongda Hospital of Southeast University and the Affiliated Suqian Hospital of Xuzhou Medical University. All patients signed informed consent forms prior to the study.

\section{Consent for publication}

Not applicable.

\section{Competing interests}

The authors declare that they have no competing interests.

\section{Author details}

'Department of Spine Surgery, Zhongda Hospital, School of Medicine, Southeast University, Nanjing 210009, Jiangsu, China. ${ }^{2}$ The Affiliated Suqian Hospital of Xuzhou Medical University, Suqian, China.

Received: 2 September 2020 Accepted: 28 October 2020

Published online: 23 November 2020

\section{References}

1. Jones EL, Heller JG, Silcox DH, et al. Cervical pedicle screws versus latera mass screws. Anatomic feasibility and biomechanical comparison. Spine (Phila Pa 1976). 1997;22:977-82

2. Ilgenfritz RM, Gandhi AA, Fredericks DC, et al. Considerations for the use of C7 crossing laminar screws in subaxial and cervicothoracic instrumentation. Spine (Phila Pa 1976). 2013;38:E199-204.

3. Klekamp JW, Ugbo JL, Heller JG, et al. Cervical transfacet versus lateral mass screws: a biomechanical comparison. J Spinal Disord. 2000;13:515-8.

4. Dunlap BJ, Karaikovic EE, Park HS, et al. Load sharing properties of cervical pedicle screw-rod constructs versus lateral mass screw-rod constructs. Eur Spine J. 2010;19:803-8.

5. Peng CW, Chou BT, Bendo JA, et al. Vertebral artery injury in cervical spine surgery: anatomical considerations management, and preventive measures. Spine J. 2009;9:70-6.

6. Park JH, Jeon SR, Roh SW, et al. The safety and accuracy of freehand pedicle screw placement in the subaxial cervical spine: a series of 45 consecutive patients. Spine (Phila Pa 1976). 2014:39:280-5.

7. Hojo $Y$, Ito $M$, Suda $K$, et al. A multicenter study on accuracy and complications of freehand placement of cervical pedicle screws under lateral fluoroscopy in different pathological conditions: CT-based evaluation of more than 1,000 screws. Eur Spine J. 2014;23:2166-74

8. Ishikawa Y, Kanemura T, Yoshida G, et al. Intraoperative, full-rotation, threedimensional image (O-arm)-based navigation system for cervical pedicle screw insertion. J Neurosurg Spine. 2011;15:472-8.

9. Abumi K, Ito M, Sudo $H$. Reconstruction of the subaxial cervical spine using pedicle screw instrumentation. Spine (Phila Pa 1976). 2012;37:E349-56.

10. Lee SH, Kim KT, Suk KS, et al. Assessment of pedicle perforation by the cervical pedicle screw placement using plain radiographs: a comparison with computed tomography. Spine (Phila Pa 1976). 2012;37:280-5.

11. Barnes AH, Eguizabal JA, Acosta FL Jr, et al. Biomechanical pullout strength and stability of the cervical artificial pedicle screw. Spine (Phila Pa 1976). 2009;34:E16-20.

12. Nakashima H, Yukawa Y, Imagama S, et al. Complications of cervical pedicle screw fixation for nontraumatic lesions: a multicenter study of 84 patients. J Neurosurg Spine. 2012;16:238-47.

13. Sucu HK, Sevin IE, Yildirim M. Central Brown-Sequard syndrome caused by hyperextension: an unexpected complication of cervical pedicle screw fixation. Clin Neurol Neurosurg. 2012;114:1003-5.
14. Yoshii T, Hirai T, Sakai K, et al. Cervical pedicle screw placement using intraoperative computed tomography imaging with a mobile scanner gantry. Eur Spine J. 2016;25:1690-7.

15. Deng $T$, Jiang $M$, Lei $Q$, et al. The accuracy and the safety of individualized 3D printing screws insertion templates for cervical screw insertion. Comput Assist Surg. 2016:21:143-9.

16. Mendelsohn D, Strelzow J, Dea N, et al. Patient and surgeon radiation exposure during spinal instrumentation using intraoperative computed tomography-based navigation. Spinal J. 2016;16:343-54.

17. Abumi $\mathrm{K}$, Itoh $\mathrm{H}$, Taneichi $\mathrm{H}$, et al. Transpedicular screw fixation for traumatic lesions of the middle and lower cervical spine: description of the techniques and preliminary report. J Spinal Disord. 1994;7:19-28.

18. Lee DH, Lee SW, Kang SJ, et al. Optimal entry points and trajectories for cervical pedicle screw placement into subaxial cervical vertebrae. Eur Spine J. 2011:20:905-11.

19. Mohi Eldin MM. Cervical pedicle screw fixation: anatomic feasibility of pedicle morphology and radiologic evaluation of the anatomical measurements. Asian Spine J. 2014;8:273-80.

20. Nishinome $M$, lizuka $H$, lizuka $Y$, et al. An analysis of the anatomic features of the cervical spine using computed tomography to select safer screw insertion techniques. Eur Spine J. 2013;22:2526-31.

21. Sugimoto $Y$, Ito $Y$, Tomioka $M$, et al. Vertebral rotation during pedicle screw insertion in patients with cervical injury. Acta Neurochir (Wien). 2010;152: 1343-6.

22. Yoshihara H, Passias PG, Errico TJ. Screw-related complications in the subaxial cervical spine with the use of lateral mass versus cervical pedicle screws: a systematic review. J Neurosurg Spine. 2013;19:614-23.

\section{Publisher's Note}

Springer Nature remains neutral with regard to jurisdictional claims in published maps and institutional affiliations.

\section{Ready to submit your research? Choose BMC and benefit from:}

- fast, convenient online submission

- thorough peer review by experienced researchers in your field

- rapid publication on acceptance

- support for research data, including large and complex data types

- gold Open Access which fosters wider collaboration and increased citations

- maximum visibility for your research: over $100 \mathrm{M}$ website views per year

At BMC, research is always in progress.

Learn more biomedcentral.com/submissions 